\title{
Large values of transverse magnetic reduction of nickel single crystal films
}

\author{
Khudoyor Urinov ${ }^{1}$, Adkham Amonov ${ }^{1}$, Khakberdi Zhumanov ${ }^{1}$, Zebo Urinova $^{2}$ and Gulam \\ Gulamov $^{3}$ \\ ${ }^{1}$ Samarkand branch of Tashkent University of information technologies named after Mukhammad al- \\ Khwarizmi, 47A Sh.Mirzo Street, Samarkand, 140100, Uzbekistan \\ ${ }^{2}$ Samarkand Institute of Veterinary and Medicine, 77 Mirzo Ulugbek Street, Samarkand, 123140, \\ Uzbekistan \\ ${ }^{3}$ Samarkand State University, 15 University avenue, Samarkand,140104, Uzbekistan
}

\begin{abstract}
In this work we studied the anisotropy of the transverse magnetoresistance of single-crystal nickel films. The measurements were carried out on samples whose surface plane coincided with the [001] cavity. Studies of magnetoresistance in a monocrystalline nickel film showed tensile stresses acting on it from the side of magnesium oxide. The modification of the magnetization anisatropy of the film on the substrates as compared with the free sample is apparently associated with a change in the shape of the Fermi surface of the carriers.
\end{abstract}

\section{Introduction}

A separate place in the physics of films is occupied by films of magnetically ordered substances (Ni, Fe, Co, and magnetic alloys). This is due to the fact that they allow us to solve a number of fundamental problems for "two-dimensional magnetism", as well as the fact that they have a number of specific magnetic properties: a specific domain structure and the associated magnetic anisotropy, "magnetization ripple", etc. [1,2]. Recently, the phenomenon of giant magnetoresistance (HMS) has been added to them, which has attracted great attention to magnetic films and is currently the subject of comprehensive research. Of particular importance is the study of the properties of magnetic films in connection with their widespread use in modern microelectronics [3-5].

The choice of Ni films as objects of study was due to a number of reasons. Firstly, Ni is an excellent candidate for studying magnetic properties depending on thickness, because has the lowest Curie point $(\mathrm{TC}=631 \mathrm{~K})$ in the series of ferromagnetic metals $(\mathrm{Fe}, \mathrm{Co})$; over the entire range of thicknesses and temperatures, $\mathrm{Ni}$, unlike $\mathrm{Fe}$ and $\mathrm{Co}$, has no polymorphic transitions, while retaining the fcc lattice responsible for ferromagnetism. With an increase in the thickness of the nickel film, both the TC dependence on the thickness and the transition from two-dimensional Ising magnets to three-dimensional Heisenberg ones are demonstrated [6,7]. Secondly, in Ni films, both in the bulk state and in the film [8], they are the simplest, as it were "model", to study their properties, in addition, to date, the electrical and especially galvanomagnetic properties of the films have been little studied.

\footnotetext{
${ }^{*}$ Corresponding author: adkham1972@gmail.com
} 
The influence of the substrate and the dependence on the angle $\varphi$ between the axis [100] of single-crystal thin nickel films on the change in the electrical resistance of films under the influence of a magnetic field or magnetization (magnetoresistance) have not been studied. Only in [2] was it reported on the technology of growing thick single-crystal films by chemical transport on a substrate of magnesium oxide. The method allows to obtain films with a thickness within $2 \mu \mathrm{m} \leq \mathrm{d} \leq 15 \mu \mathrm{m}$, and they are characterized by a relatively high ratio of residual resistances $\eta=\rho 300 \mathrm{~K} / \rho 4.2 \mathrm{~K}$, reaching 103 .

In accordance with the technology, films were grown at a substrate temperature of 10000 ${ }^{\circ} \mathrm{C}$. The coefficient of thermal expansion of nickel $\alpha \mathrm{Ni}$ exceeds the corresponding coefficient $\alpha \mathrm{MgO}$ for magnesium oxide. For example, at room temperature $\alpha \mathrm{Ni}=12.5 \cdot 10-6, \alpha \mathrm{MgO}=$ 11.2 $10-6$ deg-1. Therefore, upon cooling, the film, being bound to the substrate, undergoes a plane tensile stress, which leads to tetragonal deformation of the crystal lattice. This conclusion was subject to a comparison of the parameters of the crystal lattice measured by $\mathrm{x}$-ray diffraction at room temperature $\mathrm{T}_{\mathrm{k}}$ and $\mathrm{T}=77 \mathrm{~K}$.

The goal of this work is to study magnetoresistance and angular dependence, as well as the effect of the substrate and the dependence on the angle $\varphi$ between the [100] axis on thin single-crystal films 500-600 толщиной thick at $2950 \mathrm{~K}$ in magnetic fields up to $21 \mathrm{kOe}$.

\section{Methods of measuring magnetoresistance}

The magnetoresistance was measured by an unbalanced double bridge by Thomson with an accuracy of 10-6 Ohms. The magnetoresistance was measured in the longitudinal and transverse directions of the external magnetic field, as well as depending on the angle $\varphi(\varphi$ between the [100] axis and the direction of the external field). In all measurements, the current passed through the samples remained constant $5 \times 10-4 \mathrm{~A}$. When measuring the longitudinal and transverse magnetoresistance, the external field varied from 0 to 20,000 orested. Measurements of the effect in our experiments were carried out with an accuracy of an average of $2-4 \%$.

When measuring magnetoresistance depending on the angle $\varphi$, the value of the saturation current was 5800 Oersteds. The measurement direction r, electric current I, external magnetic field $\mathrm{H}$ with respect to the [100] axis had the following orientations: magnetoresistance for the transverse effect, when [100] $\|\mathrm{i}\| \mathrm{r} \perp \mathrm{H}$; for the longitudinal [100] $\|\mathrm{i}\| \mathrm{r} \| \mathrm{H}$. The magnetoresistance, depending on the angle $\varphi$, was measured when [100] $\|\mathrm{i}\| \mathrm{r}^{\wedge} \mathrm{H}=\varphi$.

The experimental part was performed on thin single-crystal films obtained in vacuum (10$4 \mathrm{~mm} \mathrm{Hg}$ ) on an $\mathrm{MgO}$ substrate at a substrate temperature of $5200 \mathrm{~K}$. The film thickness was $5 \mu \mathrm{m}$, the film was obtained by chemical transport reactions. They were annealed at temperatures of $300,325,350,400$, and $700^{\circ} \mathrm{C}$ in air. The deposition rate was maintained at $0.1 \mathrm{~nm} / \mathrm{s}$, and the thickness of Ni films was measured by the method of piezoelectric microweighting (quartz microbalances).

Thermal oxidation was carried out at a heating rate of $2^{\circ} \mathrm{C} / \mathrm{min}$. Once the desired maximum temperature was reached, it was maintained for 3 hours to ensure oxidation and free diffusion of $\mathrm{Ni}$ atoms on the surface. The cooling rate was set equal to $2.5^{\circ} \mathrm{C} / \mathrm{min}$.

It is assumed that the change in $\Delta \rho \rho$ with a change in the shape of the film is related to the Hall potential difference [9]. The electron diffraction pattern was used to check the plane [001] of thin nickel films.

The lead wires were fastened with a special solder consisting of clean elements: $57 \% \mathrm{Ga}$ $+23 \% \mathrm{In}+20 \% \mathrm{Sn}$ weight. parts that have a low melting point and provide reliable contact [10]. 


\section{Results and discussion}

A possible mechanism for the oxidation of a nickel film is shown schematically in Figure 1. Oxidation of nickel films occurs through the transfer of electrons, nickel cations, and oxygen anions through the film. Annealing controls the oxidation process and the morphology of the film. Photographs of Ni films on $\mathrm{MgO}$ substrates after annealing at 300, 325, 350, 400, and $700{ }^{\circ} \mathrm{C}$ are shown in Fig. 2. According to the analysis of this study, it was found that $\mathrm{Ni}$ films with a thickness of $50 \mathrm{~nm}$ are almost completely oxidized at an annealing temperature of 700 - C. The obtained results of longitudinal transverse magnetoresistance, as well as the dependence of magnetoresistance on the angle $\varphi$ for single-crystal thin films of different thicknesses are shown in Figs. 3-6. Figure 3 shows plots of the dependence $(\Delta \rho \rho)$ on $\mathrm{H}$ films obtained during the same technological cycle on an $\mathrm{MgO}$ substrate.

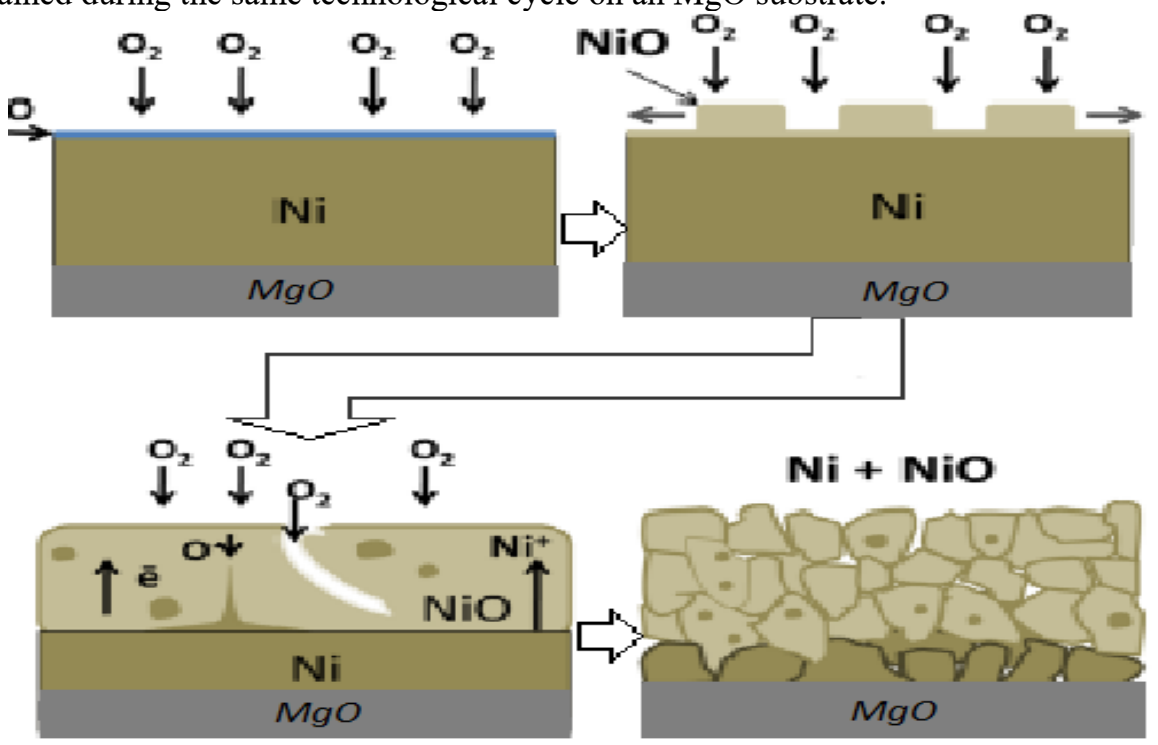

Fig. 1. Schematic representation of the process of thermal oxidation of a nickel film.

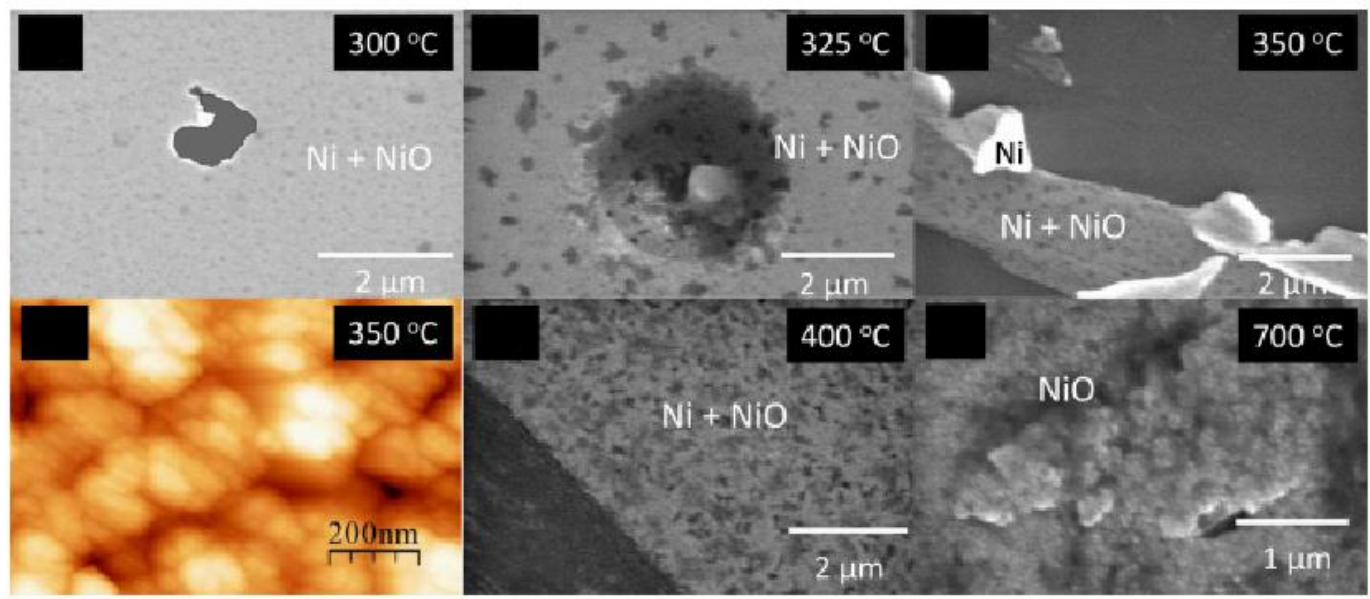

Fig. 2. Microphotographs of nickel films on $\mathrm{MgO}$ substrates at different annealing temperatures. 


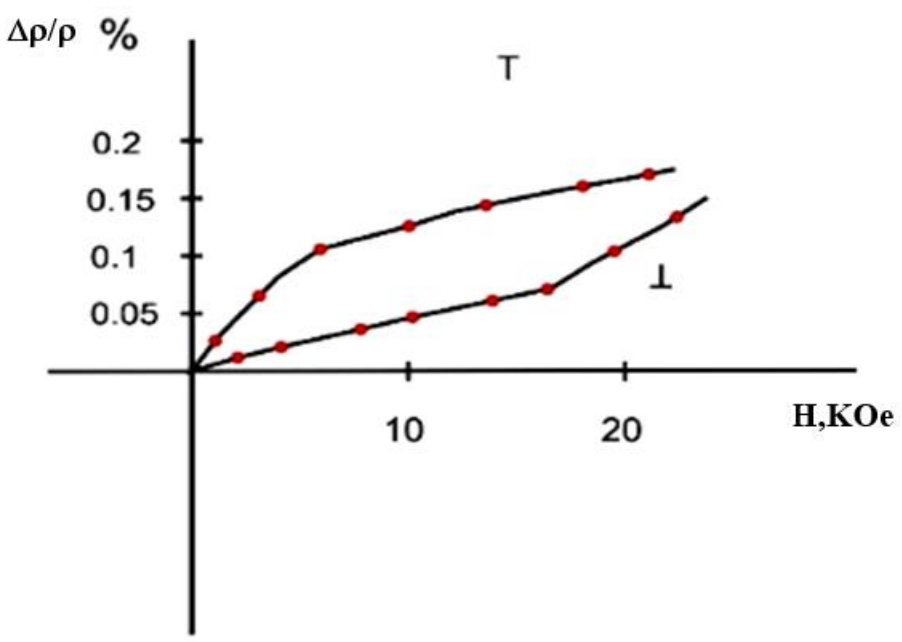

Fig. 3. Graph of the field dependence $(\Delta \rho \rho) \perp$ and $(\Delta \rho \rho) \mathrm{T}^{\top}$ of the nickel film on $\mathrm{MgO}$ substrates (film thickness $500 \AA$ ).

The direction of the current during measurements was parallel [100], the direction

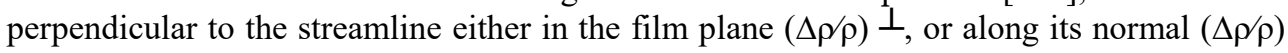
T.

The films on the $\mathrm{MgO}$ substrate experience thermoelastic stresses of different signs, which obviously correlate with the different dependences of their transverse magnetoresistance on the field $\mathrm{N}$. The $\mathrm{Ni} / \mathrm{MgO}$ film in the field of $21 \mathrm{kOe}(\Delta \rho \rho){ }_{\mathrm{T}}$ reaches $17 \%$ in $(\Delta \rho \rho) \perp_{-10 \%}$. It is assumed that tensile density stresses transfer the $\mathrm{Ni} / \mathrm{MgO}$ film to the "strong" (according to Campbell, see Fig. 4.) ferromagnet characterized by a high magnitude of magnetoresistance (up to $25 \%$ at low temperatures). A known method of converting nickel to the state of a "strong" ferromagnet is the introduction of impurities (Fe, $\mathrm{Co}, \mathrm{Cu}$, etc.) into it. The large $\Delta \rho \rho$ values for $\mathrm{Ni} / \mathrm{MgO}$ films caused by mechanical stresses are evidenced by the fact that during repeated heating - cooling cycles these values will decrease [11].

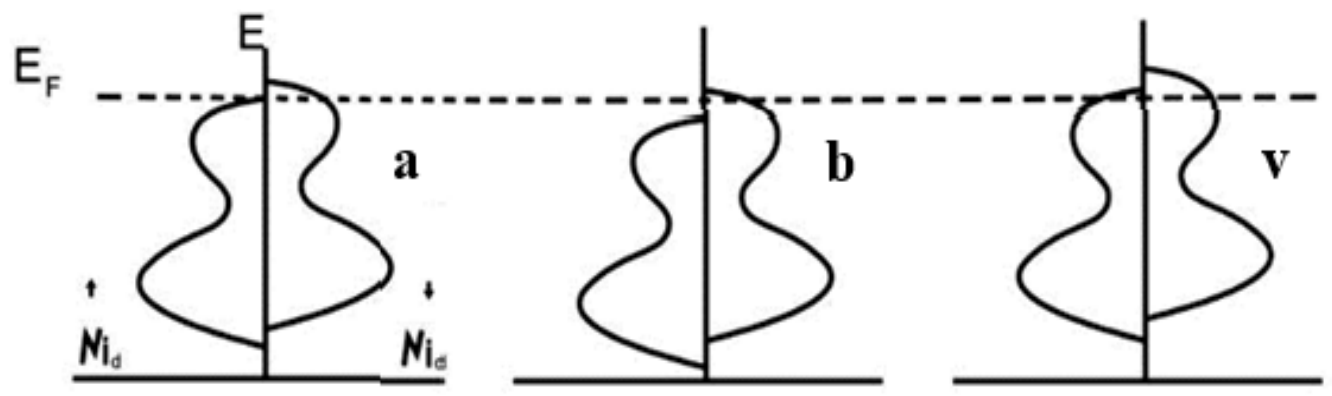

Fig. 4. Schemes of the density of states d of the nickel zone (a), "strong" (b) and "weak" (c) ferromagnets.

When measuring magnetoresistance depending on the angle $\varphi$, a shift towards the negative effect is observed with a decrease in the film thickness (Fig. 5). 


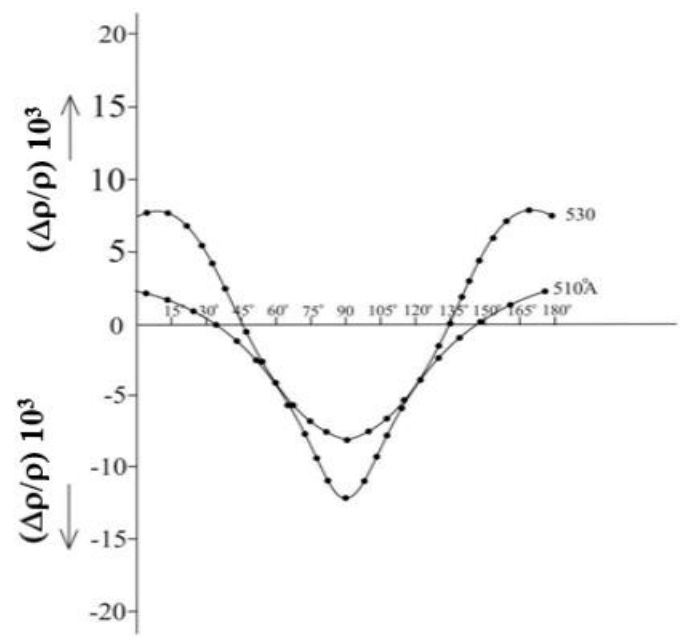

Fig. 5. The dependence of the magnetoresistance $\Delta \rho \rho$ of the angle $\varphi=[010]\|\mathrm{i}\| \mathrm{r}^{\wedge} \mathrm{H}$ at a field $\mathrm{H}=$ 5800 Oe.

The method allows to obtain films with a thickness within $2 \mu \mathrm{m} \leq \mathrm{d} \leq 15 \mu \mathrm{m}$, and they are characterized by a relatively high ratio of residual resistances $\eta=\rho 300$ to / $\rho 4,2 \mathrm{k}$, reaching 103.

In this work we studied the anisotropy of the transverse magnetoresistance of singlecrystal nickel films. The measurements were carried out on samples whose surface plane coincided with the (001) cavity. Samples with a thickness of $d=5.0 \pm 0.5 \mu \mathrm{m}$ with a ratio of $\eta=103$ were used.

Figure 6 shows the angular dependences of the transverse magnetoresistance $(\Delta \rho \rho)(\varphi)$ of such a film at $\mathrm{T}=4.2 \mathrm{~K}$ and the current direction along [110]. Here $\Delta \rho=\rho \mathrm{H}-\rho$, where $\rho \mathrm{H}, \rho$ are the resistivities of the sample in an external field $\mathrm{H}$ and in the absence of a field $(\mathrm{H}$ $=0)$. The angle $\varphi$ determines the direction of $\mathrm{H}$ relative to the normal to the plane of the film (at $\varphi=900 \mathrm{~N} \| \mathrm{n}$ ). Curves 1,2 show the dependences $(\Delta \rho \rho)(\varphi)$ for the film bonded to the substrate and, therefore, under the action of tensile stresses, at $\mathrm{H}=14$ (1) and $21 \mathrm{kOe}(2)$. We note that repeated cooling and heating of the film on the substrate from $\mathrm{Tk}$ to $\mathrm{T}=4.2 \mathrm{~K}$ did not change the dependence $(\Delta \rho \rho)(\varphi)$. Curve 3 is a graph of $(\Delta \rho \rho)(\varphi)$ at $\mathrm{H}=21 \mathrm{kOe}$ on the same film, but separated from the $\mathrm{MgO}$ substrate.

After removing the film from the substrate, the отношение ratio decreased to $\sim 870$. As can be seen from a comparison of curves 2 and 3, stress relieving leads to a noticeable change in the angular dependence of the transverse magnetoresistance of nickel films. It should be noted that at $\mathrm{T}=\mathrm{T}_{\mathrm{k}}$ and $\mathrm{T}=77 \mathrm{~K}$ the curves $(\Delta \rho \rho)(\varphi)$ obtained by us coincide with the known (3) results for the anisotropy of the magnetoresistance of bulk samples. Moreover, as it turned out, the separation of the film from the substrate does not change the dependence $(\Delta \rho \rho)(\varphi)$ at the indicated temperature values.

The angular dependence of the magnetoresistance of the film taken from the substrate at $\mathrm{T}=4.2 \mathrm{~K}$ (Fig. 6) in general terms corresponds to the graph $(\Delta \rho \rho)(\varphi)$ of the transverse magnetoresistance of the single-crystal nickel $\operatorname{rod} \eta=2700$. 


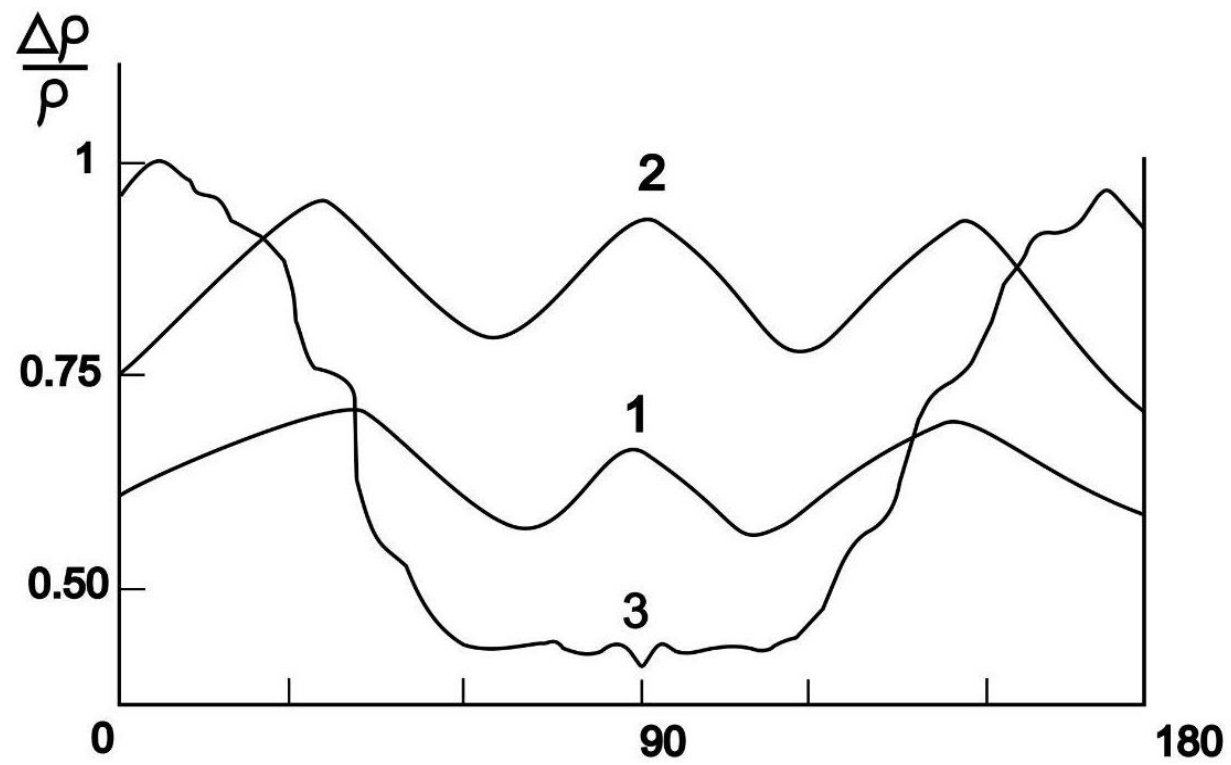

Fig. 6 Angular dependences of the transverse magnetoresistance $(\Delta \rho / \rho)(\varphi)$ of such a film at $\mathrm{T}=4.2 \mathrm{~K}$ and the current direction along [110].

The anisotropy of the magnetoresistance in this work was associated with the shape of the nickel Fermi surface. In contrast to [2], the curve we obtained does not have sharp minima for the $\mathrm{H}$ directions that coincide with the crystallographic axes of the [111] type, and the minima for $\mathrm{H} \|$ [100] do not reach the value noted by the authors [4]. Such a difference, in our opinion, is associated with electron scattering at the sample boundaries. In the magnetic field used to measure the magnetoresistance of nickel films, the cyclotron radius of conductivity electrons is $r \geq d$. Note that our data are closer to the curve $(\Delta \rho \rho)(\varphi)$ given in the work of Fossett and Reed than to the results of [5], where measurements were performed on a massive sample with $\eta=1000$. It is known the static resistance of films in a magnetic field is determined by the mean free path of electrons, lieff, to which the scattering of carriers on the film surface contributes. At the same time, the samples have electrons that do not collide with the surface and have a mean free path $1>1$ eff. Thus, electrons under the condition $1>>r$ can contribute to the magnetoresistance of the film, which is determined by the shape of the Fermi surface, which is observed experimentally.

Thus, the study of the anisotropy of the magnetoresistance of the nickel film on the $\mathrm{MgO}$ substrate allows us to conclude that the shape of the Fermi surface of the conduction electrons is caused by tetrogonal deformation of the crystal lattice of the deposited material. As far as we know, such significant changes in $\Delta \rho$ / $\rho$ under the influence of external stresses in nickel were not previously observed.

It is noteworthy that the $\Delta \rho / \rho$ value in the film on the substrate at $H \| \mathrm{n}(\varphi=900)$ is greater than at $\mathrm{H}$ in the plane of the sample, despite the lower value of induction $\mathrm{B}$ in the first case. Indeed, in a film ferromagnetic sample magnetized to saturation, at $\mathrm{H} \| \mathrm{n}$ the demagnetizing field is Nrazm $=-4 \pi \mathrm{M}$, as a result of $\mathrm{B} \approx \mathrm{H}$. When $\mathrm{H}$ in the plane of the film, $\mathrm{B}=\mathrm{H}+4 \pi \mathrm{M}$. It is obvious that a decrease in induction cannot explain the minimum in the dependence $(\Delta \rho \rho)(\varphi)$ on the film removed from the substrate at $\varphi=900$.. For the direction of the current along [100] (in this case, when $\mathrm{H}$ is in the film plane and when $\mathrm{H} \mathrm{H} \mathrm{n}$ the magnetic field is applied along equivalent crysallographic directions), a study of the dependence of $\Delta \rho \rho$ on $\mathrm{H}$ showed that in fields larger than the saturation field, the same values of $\Delta \rho \rho$ for the mentioned mutually perpendicular directions of the field are obtained for $\mathrm{H}$, differing by $6 \mathrm{kOe}$. This is approximately $4 \pi \mathrm{M}$ in nickel. The dependences $\Delta \rho \rho$ on the 
magnitude of the magnetic field in the region of $\mathrm{H}<21 \mathrm{kOe}$ for any $\varphi$ represents a monotonically rising function $\mathrm{N}$. Measurements of the dependence $\mathrm{M}(\mathrm{H})$ at $\mathrm{T}=4.2 \mathrm{~K}$ showed that the magnetic saturation of the sample along the normal is achieved in the field of $\mathrm{H} \geq 5 \mathrm{kOe}$. The exit of $\mathrm{M}$ in the direction $\mathrm{n}$ is facilitated by induced perpendicular anisotropy due to the action of tensile stresses on the film.

It is known in a number of cases, for example, when the Kolerov curves are plotted [7], it is necessary to know the resistance of a ferromagnet at $\mathrm{B}=0$. By virtue of the fact noted above that at $\mathrm{M} \| \mathrm{n}$ the magnetic induction of the film is $\mathrm{B} \approx \mathrm{H}$, the value of its resistance at $\mathrm{B}$ $=0$ is obtained by simple extrapolation of the dependence $\rho(\mathrm{H})$ obtained in the magnetic saturation region of the sample at $\mathrm{H} \| \mathrm{n}$ to the value at $\mathrm{H}=0$.Using this procedure, we obtained the dependences $(\delta \rho / \rho 0)(\mathrm{H})$, for $\mathrm{H}$ in the plane of the film and the measuring current directed along [100]. Here $\delta \rho=\rho в-\rho 0$, where $\rho$ в is the resistance value of the sample with an induction field in it equal to $\mathrm{B} ; \rho 0$-resistance at $\mathrm{B}=0$. The measurements were carried out on three samples on a $\mathrm{MgO}$ substrate, differing in $\eta$ and thickness. Two samples with $\mathrm{d}=9$ $\mu \mathrm{m}$ had respectively $\eta=800$ (sample No. 1 ) and $\eta=460$ (sample No. 2). Sample No. 3 with $\mathrm{d}=5 \mu \mathrm{m}$ had $\eta=380$. Measurements $(\delta \rho / \rho 0)(\mathrm{H})$ on samples No. 2, 3 were repeated after separating them from the substrate.

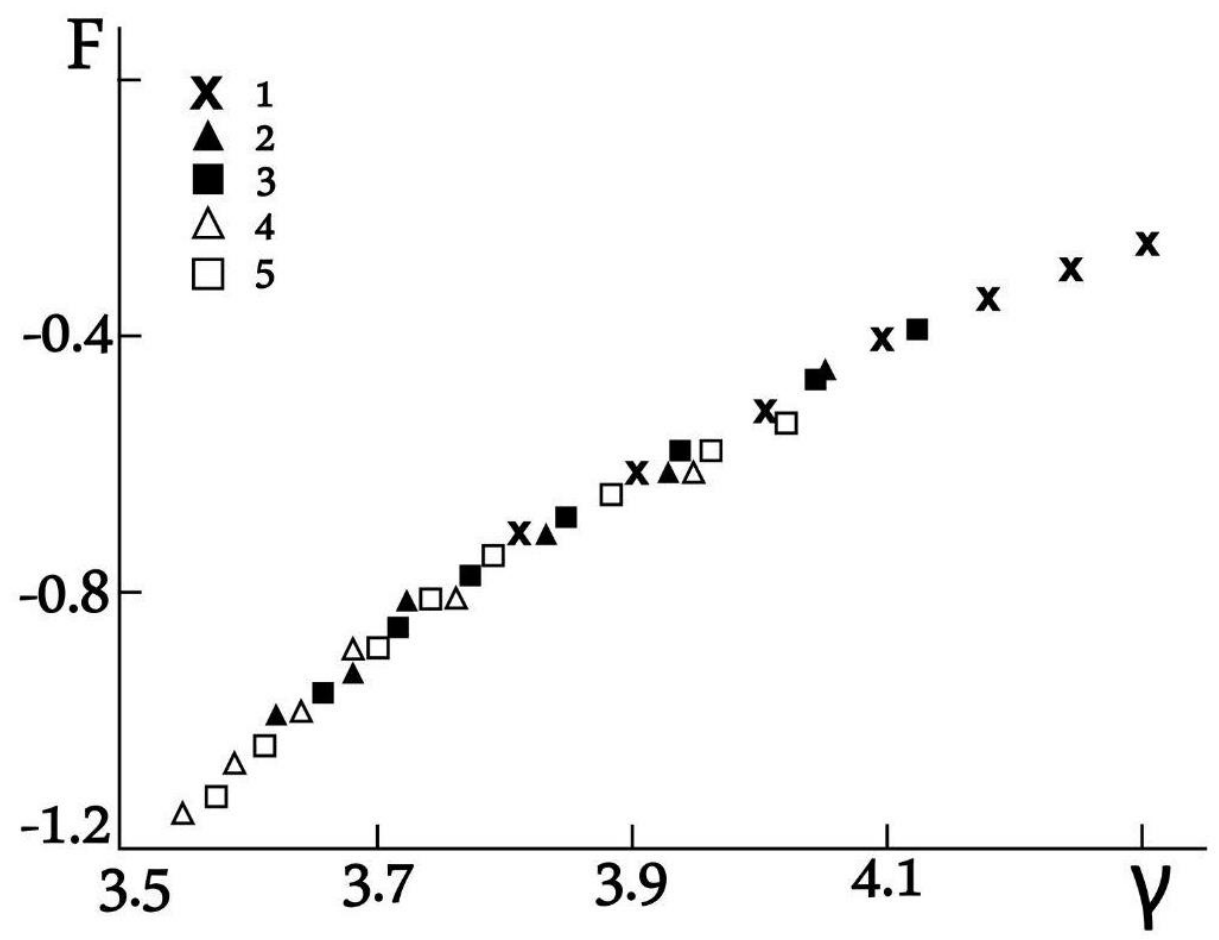

Fig. 7. Dependences $F=\log (\Delta \rho / \rho)$ on $\gamma=\log (B \eta d * / d)$ of single-crystal nickel films: 1-3 films on a substrate; 4, 5-films separated from the substrate. $d(\mu \mathrm{m}), \mathrm{n}: 1-9,800 ; 2-380 ; 3-9,460 ; 4-5,250 ; 5-$ 9,380 .

In Figure 7 shows the universal dependence, $\delta \rho / \rho=\mathrm{f}(\mathrm{B \eta d} * / \mathrm{d})$, which obeys the magnetoresistance of the studied samples; here $\mathrm{d}^{*}=9 \mu \mathrm{m}$. Moreover, the points obtained by measuring individual points from the average course of universal credit do not exceed $10 \%$. The ability to imagine the magnetoresistance of a universal curve depending on the thickness of the sample indicates the presence of a size effect, the existence of which was assumed when discussing the results of studying the anisotropy of magnetoresistance. 
Thus, the effect of tensile stresses acting on it from the side of magnesium oxide on the alternating magnetoresistance of a monocrystalline nickel film is demonstrated.

\section{Conclusions}

The modification of the anisatropy of the magnetoconductivity of the film on the substrate as compared with a free sample is associated with a change in the shape of the Fermi surface of carriers. In this case, the volume occupied by carriers in the momentum space does not change, as indicated by the existence of a common universal dependence for the magnetoresistance of films on a substrate and films separated from it.

The magnetoresistance in both the longitudinal and transverse magnetic fields for all the studied film thicknesses has a different curve: in the first case with a positive, in the second with a negative value of the effect.

The magnitude of the magnetoresistance with a magnetic saturation field of $5800 \mathrm{Oe}$, depending on the angle $\varphi$ between the [100] axis and magnetic field $\mathrm{H}$, shifts to the negative effect with decreasing film thickness and reaches zero at $\varphi$ equal to 1450 and 1350 to 600 films $\AA$.

On films with a thickness of $500 \AA$, the magnitude of the magnetoresistance in the entire range of the angle change has a negative sign of the magnitude of the effect.

With a decrease in temperature, after the transition of the $\mathrm{Ni} / \mathrm{MgO}$ film, a "strongly magnetic" state occurs in it a structural phase transition.

\section{References}

1. P. Poulopoulos, K. Baberschke, J. Phys. Condens. Matter, 11 (1999)

2. G. I. Frolov, J. Technical Physics, 12 (2001)

3. F. Huang, M.T. Kief, G.J. Mankey J. Phys, Rev, 6 (1994)

4. O. V. Snigirev, A. M. Tishin, S. A. Gudoshnikov, J. Sol. Stat. Phys, 9 (1998)

5. V. B. Loboda, S. M. Pirogov, C. I. Protsenko, Bulletin of SSU. Series Physics, Mathematics, Mechanics, 3-4 (2001)

6. V. B. Loboda, S. M. Pirogov, Yu. O. Shkurkoda Bulletin of SSU. Series Physics, Mathematics, Mechanics, 13 (2002)

7. M. Viret, D. Vignoles, D. Cole J. Phys. Rev, 53 (1996)

8. P. D. Kim, D. L. Khalyapin, I. A. Turpanov, J. Solid, State Physics, 9 (2000)

9. S. V. Kan, N. I. Kiselev, Yu. I. Mankov, J. Physics of Met. and Metall, 3 (1987)

10. Kh. O. Urinov, Kh. A. Zhumanov, A. M. Khidirov, Kh. B. Mirzokulov, Zh. O. Urinov, Scientific Bulletin of Samarkand State University, 3 (2019)

11. Kh. O. Urinov, A. N. Salakhitdinov, Kh. B. Mirzokulov Bulletin of the Russian Academy of Sciences: Physics, 83, 7 (2018) 